\title{
Role of Humoral and Cell-Mediated Immune Responses against Influenza Infections
}

\author{
Hawa Vahed ${ }^{1}$, Mirza Naqi Zafar $^{2}$ and Nusrat Jamil ${ }^{1 *}$ \\ ${ }^{1}$ Department of Microbiology, University of Karachi, Karachi, Pakistan \\ ${ }^{2}$ Sindh Institute of Urology and Transplantation, Karachi, Pakistan \\ *Corresponding author: Jamil N, Department of Microbiology, University of Karachi, Karachi, Pakistan, Tel: 923002162465; E-mail: nusrat_91@yahoo.com
}

Received date: October 25, 2016; Accepted date: January 09, 2017; Published date: January 16, 2017

Copyright: (c) 2017 Vahed $\mathrm{H}$ et al. This is an open-access article distributed under the terms of the Creative Commons Attribution License, which permits unrestricted use, distribution, and reproduction in any medium, provided the original author and source are credited.

\begin{abstract}
This study was designed to determine the extent of exposure of individuals to the avian influenza viruses by evaluating their humoral and cell-mediated immune response. Therefore blood samples were collected from three cohorts i.e. poultry related personals, healthy individuals and flu patients. Serum and PBMC analysis provided the information about humoral and cell-mediated immune responses respectively. The serum samples were examined for the presence of antibodies against indigenous avian influenza strains by using vaccines i.e. avian influenza (H5) monovalent vaccine, avian influenza $(\mathrm{H} 7+\mathrm{H} 9)$ bivalent vaccine and commercially available four human international brands of Bird/Swine influenza vaccines i.e. Aventis 2008/2009, Vaxigrip 2009/2010, Agripal 2009/2010 and Vaxigrip $2010 / 2011$ by Dot-Blot assay. In parallel the lymphocytes were isolated from the collected blood of these cohorts and cell mediated immune response was determined by ELI-spot assay and HLA subtyping. The ELI-spot assay of 50 samples of lymphocytes revealed the positive spot for Influenza virus subtype H9. $90 \%$ and $70 \%$ of the lymphocytes were positive for influenza virus subtype $\mathrm{H} 7$ and $\mathrm{H} 5$ respectively. Interestingly, it has been concluded from the results that all those individuals who were having antibodies against $\mathrm{H} 5, \mathrm{H} 7$ and $\mathrm{H} 9$ were also positive for ELI-spot assay and they never being immunized against influenza viral strains. Whereas HLA results in chicken hawkers and flu patients revealed that MHC Class II alleles are majorly involved in the susceptibility and resistance to influenza infections. Results indicated that majority of the individuals were heterozygous at all HLA Loci, homozygous individuals at MHC Class II loci showed a highly significant association with influenza viral infection. Whereas the Homozygosis at HLA-class I loci A and B had no effect on the rate of disease progression. Such immunogenetical studies will help to develop an enhanced mechanistic understanding of the immune response to seasonal flu responses as well.
\end{abstract}

Keywords: Humoral immune response; Cell-mediated immune response; PBMCs; Influenza virus

\section{Introduction}

Zoonoses refer to the infectious animal diseases that are communicable to humans [1]. Zoonotic disease can be determined by comparing the antibodies in a human population that has a close association with animals, to the antibody level of a general population with little or no animal association [2]. The postulate under which this study was done was to investigate dissemination of avian zoonotic viral infections i.e. avian influenza from stray birds and open poultry markets into poultry related persons (chicken hawkers) and healthy individuals.

Influenza viruses constitute a serious public health problem, causing severe illness and death within high-risk populations during seasonal epidemics and, more rarely but recurrently, influenza A virus related pandemics [3]. Over 100 million poultry birds have been affected by this destructive disease in last two years worldwide [4]. Some of the avian influenza A strains are common etiology for bird infections in Pakistan. Although their transmission and fatality to human has not been reported but human exposure to these viruses cannot be ruled out.

In recent times, great interest has been generated in genetic markers that interact with other factors for determining host's responses to the infectious diseases [5,6]. Genetic factors are among the main determinants for susceptibility to infectious diseases in human beings $[5,7]$. Epidemiological studies have demonstrated that more than 40 diseases are more frequently found in individuals carrying certain types of HLA class I or II alleles in comparison to other general populations [8]. The magnitude of these effects could be quite large but probably not be absolute [9]. HLA is the most polymorphic genetic system in human beings having numerous alleles and consequently have the combinations with different possibilities [5].

Human major histocompatibility complex (MHC) genes are located on the short arm of chromosome 6. MHC contains the most polymorphic loci in human beings, named as the human leukocyte antigens (HLA) [10,11]. MHC are divided into three classes i.e. I, II and III. Humans have three major MHC class I genes which are known as the HLA-A, HLA-B and HLA-C [12]. MHC class I region is occupied by genes primarily encoding for proteins that present intracytoplasmic (e.g. viral) antigens for the activation of cytotoxic Tcells (CD8+), MHC class II region consists of genes responsible for presentation of phagocytosed antigens from extracellular pathogens to helper T-cells (CD4+). Whereas, MHC class III region contains genes with a functional role in innate (non-specific) immunity, inflammation, and regulation of the immune response [13].

By defining the protective HLA allelic associations, potentially allow the identification of pathogen epitopes which are restricted by specific HLA alleles. Such epitopes can be incorporated into vaccine designs 
with the expectation that resistance they exhibit would be replicated by immunization in particular if any of the key mechanism of the immunological protection has HLA-restricted CTL response. Such experimental approaches are termed as "reverse immunogenetics," and have been attempted for a number of infectious diseases where the protective HLA associations are described $[14,15]$.

\section{Materials and Methods}

Detection of humoral immune response in chicken hawkers, healthy individuals and flu patients against influenza viruses by Dot-Blot assay

Questionnaire form was designed during this study and given to the volunteers for their consents for participation. The participants who were willing to participate in the study came by themselves, and were free to cease the participation any time but they contributed willingly for the piece of research in which their participation was required.

Four seasonal human influenza vaccines including, Aventis 2008/2009, Vaxigrip 2009/2010, Agripal 2009/2010 and Vaxigrip 2010/2011 were bought from the pharmacy store in Karachi (which is locally available for the human population to protect themselves from the circulating strains of Flu). The vaccines were kept refrigerated at $4^{\circ} \mathrm{C}$ until used (as mentioned in the package).

Crude preparation of antigens was used to develop Dot-Blot Assay. Briefly, the avian influenza vaccines including $\mathrm{H} 5$ monovalent vaccine, and $\mathrm{H} 7+\mathrm{H} 9$ bivalent vaccine and sterile allantoic fluid (as control) were separately diluted by adding $25 \%$ deionized distilled water, proteins were denatured by adding $12.5 \% \beta$-mercaptoethanol $(\mathrm{v} / \mathrm{v})$ and heated for 10 minutes at $100^{\circ} \mathrm{C}$. Four seasonal human influenza vaccines including, Aventis 2008/2009, Vaxigrip 2009/2010, Agripal $2009 / 2010$ and Vaxigrip 2010/2011 were mixed separately with equal volume of $\beta$-mercaptoethanol $(3 \mu \mathrm{l})$ and heated for 10 minutes at $100^{\circ} \mathrm{C}$ for the denaturation of proteins.

To perform Dot-Blot assay samples were immobilized on to the nylon membrane according to the labelled grid. The membrane was allowed to air dry, transferred into the blocking buffer, containing $6 \%$ non-fat milk in TBST, incubated for 2 hours at room temperature with intermittent shaking. After incubation, the membrane was washed twice with TBST and transferred to diluted antiserum i.e. primary antibodies against avian influenza (H5 and H7) and swine influenza subtypes (1:500) separately, incubated for 2 hours at room temperature with regular shaking. Afterwards membrane was washed six times with TBST. The anti-human IgG $\gamma$-chain specific alkaline phosphatase conjugate (secondary antibodies) were diluted 1:2500 in TBST and membrane was placed in it under constant shaking conditions for 30 minutes at room temperature. The membrane was washed six times with TBST then $5 \mathrm{ml}$ substrate solution (BCIP/NBT tablet dissolved in $10 \mathrm{ml}$ of $\mathrm{D} / \mathrm{W}$ ) was added and kept shaking till sufficient color was developed.

\section{Detection of humoral immune response in chicken hawkers against influenza viruses by ELISA}

Antigen (the indigenous avian viral vaccines and four human international brand of Swine influenza vaccine) was diluted in coating buffer to a concentration of $0.2 \mathrm{~mol} / \mathrm{L}$, and $50 \mu \mathrm{l}$ per well was added to the 96-well flat bottom plate. Following an overnight incubation at $4^{\circ} \mathrm{C}$, the wells were washed thrice with washing buffer $(0.05 \%$ Tween
20 in PBS) and subsequently blocked by blocking buffer (4\% non-fat milk in TBST) for 1 hour at $37^{\circ} \mathrm{C}$. Blocking buffer was discarded and the plates were dried on paper towel. Plates were either used immediately or stored at $-80^{\circ} \mathrm{C}$ until use.

Chicken hawkers sera (primary antibodies) were diluted 1:1000 in sterile deionized distill water, $50 \mu \mathrm{l}$ per well was added in respective well and the plate was incubated for 30 minutes at $37^{\circ} \mathrm{C}$. Subsequently, the plate was washed five times with washing buffer. Anti-human-IgGspecific alkaline phosphatase conjugate (secondary antibodies) were diluted 1:2000 in sterile PBS, and $50 \mu \mathrm{L}$ per well was added in the wells and incubated for 30 minutes at $37^{\circ} \mathrm{C}$.

Following 5 times washes with washing buffer, color was developed by adding $50 \mu \mathrm{l}$ per well of (BCIP/NBT tablet dissolved in $10 \mathrm{ml}$ of $\mathrm{D} / \mathrm{W}$ ) substrate solution. After 10 minutes of incubation at $37^{\circ} \mathrm{C}$, the color reaction was stopped with an equal volume of stop reagent i.e., 2 $\mathrm{mol} / \mathrm{L}$ sulfuric acid to each well. The intensity of the reaction was photo-metrically measured at $450 \mathrm{~nm}$ with an automated ELISA Reader.

\section{Detection of cell-mediated immune response in chicken hawkers, healthy individuals and flu patients against influenza viruses by ELI-spot assay of lymphocytes}

Lymphocytes were isolated from $5 \mathrm{ml}$ blood collected from chicken hawkers, healthy individuals and flu patients in EDTA containing tubes. In separate $15 \mathrm{ml}$ Falcon tubes, $5 \mathrm{ml}$ of RPMI-1640 culture medium (without FBS) was mixed with equal volumes of blood. $10 \mathrm{ml}$ ficoll paque plus was taken in another $15 \mathrm{ml}$ Falcon tube and the diluted blood was layered on it carefully, spun at 1800 RPM for 20 minutes at room temperature. PBMCs were appeared at the junction of two layers. The upper layer was discarded and the PBMCs were transferred to another sterile tube. The PBMCs were washed with RPMI-1640 medium (without FBS) and spun at 1800 RPM for 20 minutes at $4{ }^{\circ} \mathrm{C}$. The pellet was re-suspended in $1 \mathrm{ml}$ RPMI-1640 (with FBS) and used for Eli-spot assay and DNA extraction.

ELI-spot Assay was performed by using $1 \times 10^{5}$ lymphocytes, diluted in five times coating buffer and immobilized on to the nylon membrane according to the labelled grid. The membrane was allowed to air dry then transferred into the blocking buffer ( $4 \%$ non-fat milk in PBST) and incubated for 2 hours at $37^{\circ} \mathrm{C}$ with intermittent shaking. The membrane was washed twice with washing buffer (PBST) then transferred to diluted antiserum i.e. primary antibodies (1:1000) and incubated for 30 minutes at $37^{\circ} \mathrm{C}$ with regular shaking and wash six times with washing buffer.

The anti-chicken antibodies conjugated with horse reddish peroxidase i.e. secondary antibodies were diluted 1:2000 in distill water and the membrane was placed in it under constant shaking for 30 minutes at $37^{\circ} \mathrm{C}$. The membrane was washed six times with washing buffer then $5 \mathrm{ml}$ substrate TMB-hydrogen peroxide (3', 5', 3', 5'tetramethylbenzidine-hydrogen peroxide) was added and kept in shaking till sufficient color was developed.

\section{Detection of cell-mediated immune response in chicken hawkers by human leucocytes antigen (HLA) subtyping by CTS-PCR-SSP method}

DNA was isolated from PBMCs by using $10 \mathrm{mg} / \mathrm{ml}$ proteinase $\mathrm{K}$ enzyme and 5 times proteinase $\mathrm{K}$ buffer, SDS $10 \%$ and deionized $\mathrm{d} / \mathrm{w}$. Digested sample was vortex and $6 \mathrm{M} \mathrm{NaCl}$ was added. The sample was 
vortex again until the solution turned milky and then spun at 13,000 rpm for 5 minutes at room temperature. The supernatant was precipitated with $1 \mathrm{ml}$ of absolute ethanol (chilled at $-20^{\circ} \mathrm{C}$ ) and pelleted by spinning at $14,000 \mathrm{rpm}$ for 5 minutes. The pellet was washed with $1 \mathrm{ml}$ of $70 \%$ ethanol (chilled at $-20^{\circ} \mathrm{C}$ ), vortex and spun at 13,000 rpm for 2 minutes. Supernatant was discarded and DNA pallet was dried on heating block. Deionized distill water $(100 \mu \mathrm{l})$ was added in the dried pellet and vortex in order to dissolve the DNA. DNA was preceded for the concentration and purity by determining the OD 260/OD 280 ratio.

PCR Amplification for HLA subtyping of the samples was performed by using CTS-PCR-SSP tray kit, containing 141 primer mixture sequences. Reaction mix contained master mix, Taq DNA polymerase, approximate $0.1-0.15 \mu \mathrm{g} / \mathrm{ml} \mathrm{DNA}$ and deionized distill water (as mentioned by manufacturers) mixed well and $10 \mu \mathrm{l}$ was added to each well of the CTS-PCR-SSP tray kit containing 141 primer mixture sequences separately. The PCR conditions used were initial denaturation at $92^{\circ} \mathrm{C}$ for 2 minutes, denaturation at $94^{\circ} \mathrm{C}$ for 15 seconds and annealing+extension at $65^{\circ} \mathrm{C}$ for 1 minutes for 10 cycles, followed by 10 cycles of denaturation at $94^{\circ} \mathrm{C}$ for 15 seconds, annealing at $61^{\circ} \mathrm{C}$ for 50 seconds, and extension at $72^{\circ} \mathrm{C}$ for 30 seconds. Amplified products were analyzed on $2 \%$ agarose gel electrophoresis and DNA profile was noted.

\section{Results}

Detection of humoral immune response in chicken hawkers, healthy individuals and flu patients against influenza viruses by Dot-blot assay and ELISA

In the present study, antibodies were detected against influenza viruses by Dot-Blot assay. The ELISA based Dot-Blot (immunebinding) assay was designed by immobilizing antigens directly onto a nitrocellulose membrane, it is an alternative to ELISA and possesses distinct advantages, including direct visualization of the spots on the membrane. The cohort consisted of 50 sera samples from poultry related personals (Chicken hawkers) $n=14$ ranging from 21-52 years of age collected during December 2012, sera from Flu patients $n=17$ ranging from 02-50 years of age were collected during 2012 and sera from healthy individuals $n=19$ ranging from $20-30$ years of age were collected in 2012. The results of Dot-Blot assay demonstrated that 58\% sera samples detected the presence of antibodies against avian influenza subtypes $\mathrm{H} 5$. Whereas $87 \%$ sera samples were found to be positive for the mixture of $\mathrm{H} 7+\mathrm{H} 9$ strains of avian influenza strains included in domestic vaccine. Interestingly, $96 \%$ and $97 \%$ sera samples of all cohorts were strongly reactive with 2 human Flu vaccines (imported from developed countries) i.e. Vaxigrip 2009/2010 and Vaxigrip 2010/2011 respectively whereas Aventis 2008/2009, Agripal $2009 / 2010$ was found to be $82 \%$ and $76 \%$ reactive with sera samples of all the cohorts (Figure 1 and Figure 2).

To confirm the dot blot assay results ELISA was also performed of chicken hawkers sera and analysed against above mentioned vaccines. The results indicated that the same samples which were positive by Dot blot assay were also positive by ELISA (Figure 3).

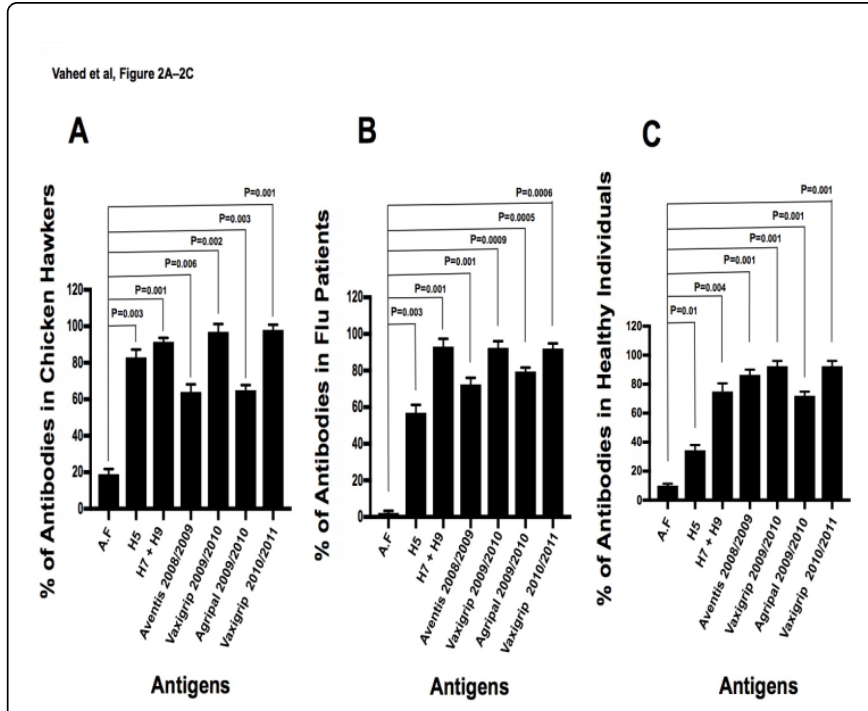

Figure 1: Screening of Chicken Hawkers, Flu patients and Healthy Individuals against Avian Influenza and Swine Influenza Viruses by Dot-Blot Assay. 50 sera samples from poultry related personals (Chicken hawkers) $\mathrm{n}=14$ ranging from 21-52 years of age collected during December 2012, sera from Flu patients $n=17$ ranging from 02-50 years of age were collected during 2009-2011 and sera from healthy individuals $n=19$ ranging from $20-30$ years were screened for the presence of antibodies against avian influenza vaccines and four human seasonal influenza vaccines by Dot Blot Assay as described in Materials and Methods. Graphs show that all the sera samples have the antibodies against different avian and swine influenza viruses. A: Humoral immune response in chicken hawkers against avian and swine influenza viruses by Dot-Blot assay. B: Humoral immune response in Flu patients against avian and swine influenza viruses by Dot-Blot assay. C: Humoral immune response in healthy individuals against avian and swine influenza viruses by Dot-Blot assay.

\section{Detection of cell-mediated immune response in chicken hawkers, healthy individuals and flu patients against influenza viruses by ELI-spot assay}

Influenza is the most contagious virus among other avian viruses and can cause severe infections in humans. Therefore cell mediated immune response was also investigated among healthy individuals, chicken hawkers and flu patients against three different strains of influenza i.e. H5, H7 and H9. The results of 50 lymphocytes samples indicated that $100 \%$ lymphocytes showed positive spot for influenza virus subtype $\mathrm{H} 9$. Whereas $90 \%$ and $70 \%$ lymphocytes were positive for influenza virus subtype $\mathrm{H} 7$ and $\mathrm{H} 5$ respectively (Figure 4).

\section{Detection of cell-mediated immune response in chicken hawkers by human leucocytes antigen (HLA) subtyping by CTS-PCR-SSP method}

The gene polymorphism of MHC loci of individuals including poultry hawkers $(n=13)$ and Flu patients $(n=02)$ against influenza strains, was determined by genotyping of the HLA Class I and II including A, B DR and DQB1. The results indicated the HLA homozygosis at the MHC class II for both DQB1 and DR alleles, 
including, poultry hawkers $(n=4)$ and Flu patient $(n=1)$ showing a highly significant association of loci with the influenza infection (Table 1) as having high level of antibodies against H5, H7, H9 and Vaxigrip 2009/2010 containing A/Brisbane/59/2007(H1N1)-souche, A/ Brisbane/10/2007(H2N2)-souche, B/Brisbane/60/2008-souche. Whereas, homozygosis at HLA-class I loci A and B had no effect on the rate of disease progression. These findings indicate an important role of the products of the class II loci in the immune response to influenza (Table 1). This study demonstrates that MHC Class II alleles are majorly involved in the susceptibility and resistance to influenza infections (Table 1).

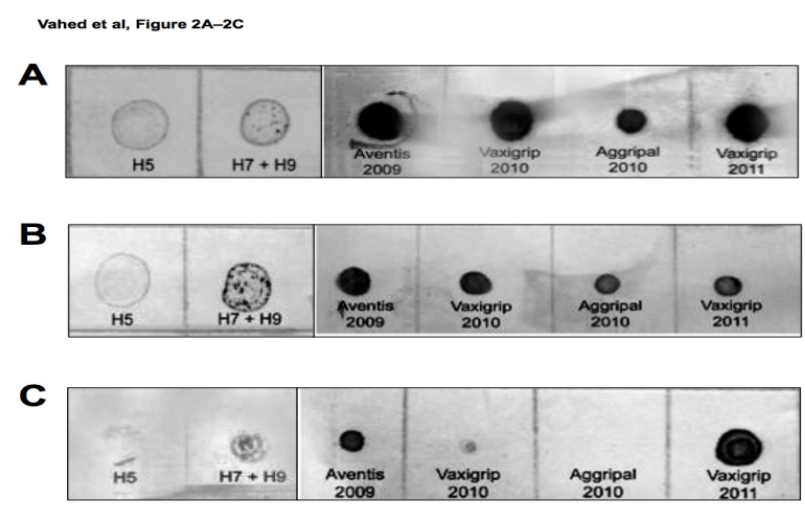

Figure 2: Humoral Immune Response in Individuals against Avian and Swine Influenza Viruses by Dot-Blot Assay. Sera samples were screened for the presence of antibodies against avian influenza vaccine and four seasonal human influenza vaccines by Dot Blot Assay as described in Materials and Methods. Antibodies were detected by blotting the antigens onto nitrocellulose membrane. The blots were immersed into diluted serum. After proper washing, blots were then immersed in conjugate (anti-human IgG $\gamma$-chain specific alkaline phosphatase). Blots were washed and spots were developed by adding chromogenic substrate (BCIP/NBT). Dots show the presence of antibodies and intensity of color is proportional to the amount of antibodies in the sera. A: Dot Blot Assay with chicken hawker serum B: Dot Blot Assay with Flu Patient serum C: Dot Blot Assay with Healthy Person serum.

\section{Discussion}

The study was designed to investigate the host's humoral and cellmediated immune response in healthy individuals, chicken hawkers, and flu patients against influenza viruses to determine the extent of exposure to these agents. In Pakistan, poultry birds are largely sold through open poultry markets. These markets play a crucial role in maintenance, amplification, and dissemination of avian influenza subtypes and are high-risk locations for potential zoonotic transmission of avian viruses to the humans. Chicken hawkers are considered as the reference index for avian viruses due to high exposure rate, to birds and their infected material as these poultry related personals have been handling the poultry birds. Transmission of avian viruses is thought to occur via direct contact with infected poultry including both the live and slaughtered birds, or surfaces and objects contaminated by their feces, blood, feathers and/or meat. In our community, individuals are in close contact with live poultry markets as well as with stray birds. Stray birds disperse bird droppings and feathers which could be the source for avian infections. Flu patients were also included in this study to identify and evaluate their etiology and immune status respectively against avian influenza virus. Therefore, a serological survey of cohorts of poultry related personals, Flu patients and a group of healthy individuals was conducted from individuals in Karachi, Pakistan by Dot-Blot assay.

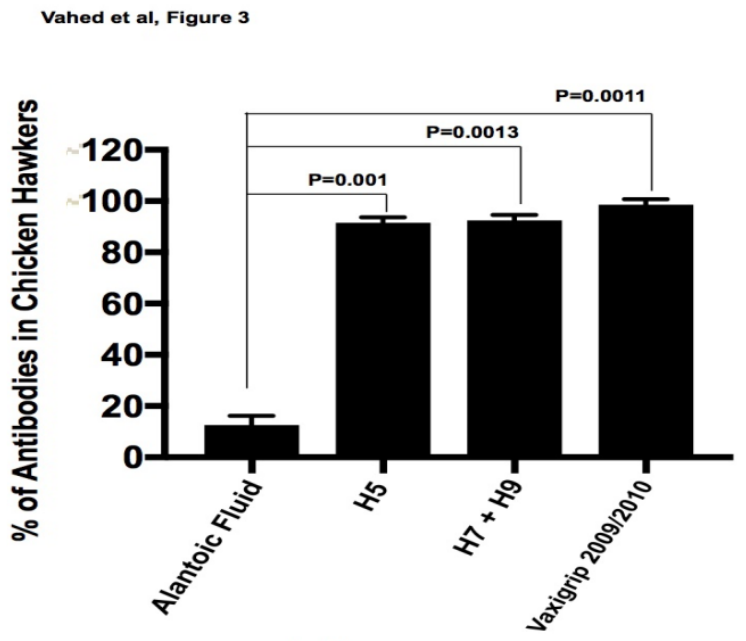

Antigens

Figure 3: Humoral Immune Response in poultry related personals (Chicken Hawkers) against Avian and Swine Influenza Virus by ELISA. 14 sera samples from poultry related personals (Chicken hawkers) ranging from 21-52 years of age were screened for the presence of antibodies against avian influenza vaccines and a seasonal influenza vaccines for humans by ELISA as described in Materials and Methods. Antibodies were detected by coating the antigens on the 96 well plates. The diluted serum was added following blocking the antigens. After proper washing, anti-human $\operatorname{IgG} \gamma$-chain specific alkaline phosphatase conjugate was added. Finally, the wells were washed and color was developed by adding chromogenic substrate (BCIP/NBT). The intensity of the reaction was photo-metrically measured at $450 \mathrm{~nm}$ with an automated ELISA Reader. Graphs show that all the sera samples have the antibodies against avian and swine influenza viruses.

This study demonstrated a promising indication of epidemiological status of the avian influenza viruses for the year 2009-2011 in the community. Presence of antibodies in most of the individuals of all the cohorts against influenza viral strains incorporated in the domestic vaccines has been noticed. These results explicitly elucidated the persistent circulation of these viral strains (Figure 1). Similar results were observed by ELISA as well, when chicken hawkers sera were analyzed against above mentioned vaccines with minor difference in their titer (Figure 3). The antibodies development in chicken hawkers is probably due to their exposure to the live poultry. Whereas, the majority of the individuals including flu patients $(n=10)$ or healthy persons $(n=17)$ were residing close to the open markets. Therefore this study suggests that the humoral immune response in most of the individuals was due to the high exposure rate of viral strains circulating in the environment close to these markets. 
However, a time response elevation in the status of immunity against subtypes of swine influenza strains included in the vaccines (imported from developed countries) has been noticed, representing that these viruses were introduced into our community in 2009 or earlier. The comparative analysis of the immunity profile of (Figure 1) all cohorts of the study reflected the recent past history of the influenza subtypes i.e. $\mathrm{H} 5, \mathrm{H} 1, \mathrm{H} 2$ and or $\mathrm{H} 3$. Interestingly, sera of all the chicken hawkers and 95\% Flu patients and healthy individuals indicated the presence of antibodies against these strains included in the SIV vaccines i.e. Vaxigrip 2009/2010 containing A/Brisbane/ 59/2007(H1N1)-souche, A/Brisbane/10/2007 (H2N2)-souche , B/ Brisbane/60/2008-souche Whereas, $100 \%$ chicken hawkers, and $94 \%$ Flu patients and $95 \%$ healthy individuals revealed the existence of antibodies against the strains included in Vaxigrip 2010/2011 containing A/California/7/2009(H1NI)-derived strain, A/ Perth/ $16 / 2009$ (H3N2) like strain, $\mathrm{B} /$ Brisbane/60/2008. This has exhibited the existence of these viral strains overall (Figure 1). It is important to note that these individuals were never being immunized with any of the mentioned vaccines therefore it can be deduced that immunity against these subtypes of avian influenza indicates the presence of these strains in our community.

\section{Vahed et al, Figure 4}

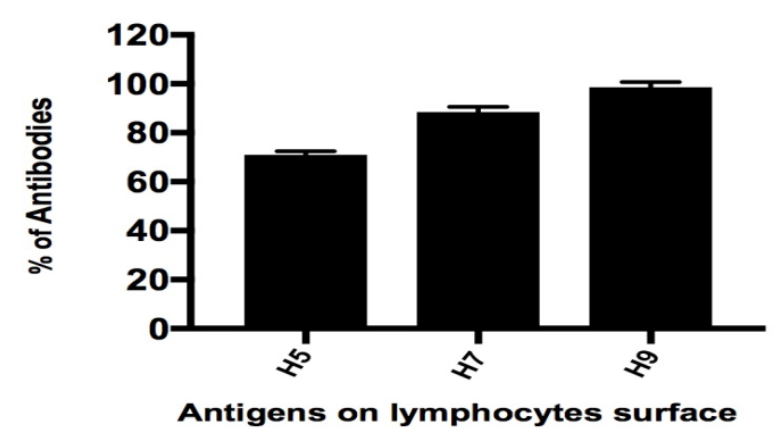

Figure 4: Cell-Mediated Immune Response in poultry related personals (Chicken Hawkers), Flu Patients and Healthy Individuals against Avian Viruses by ELI-Spot Assay. Lymphocytes from poultry related personals (Chicken hawkers), flu patients and healthy individuals were screened for the presence of virus specific antigens on the surface of circulating blood lymphocytes by ELI-spot assay as described in Materials and Methods. The Lymphocytes were immobilized on to the nylon membrane according to the labelled grid. The blots were immersed into diluted serum (H5, H7 and H9 respectively). After proper washing, blots were then immersed in conjugate (anti-chicken antibodies conjugated with horse reddish peroxidase). Blots were washed and spots were developed by adding chromogenic substrate TMB-hydrogen peroxide $\left(3^{\prime}, 5^{\prime}, 3^{\prime}, 5^{\prime}-\right.$ tetramethylbenzidine-hydrogen peroxide. Dots show the presence of antibodies and intensity of color is proportional to the amount of antibodies in the sera. Graphs show that all the lymphocyte samples were positive for more than one influenza subtype.
According to the WHO reports the global incidences of bird infections and bird Flu with newly evolved subtype H5N1 initiated in 1996 in China [16]. In present study the comparative analysis of immunity against $\mathrm{H} 7, \mathrm{H} 9$ and $\mathrm{H} 5$ in cohorts strongly supported the findings of the reports. As the elevated immunity level against $\mathrm{H} 7$ and $\mathrm{H} 9$ as compare to $\mathrm{H} 5$ immunity level (Figure 1) strongly explained the status of subtype as $\mathrm{H} 7$ and $\mathrm{H} 9$ persistence and H5 (new entry) circulating in the community this notion was further confirmed by ELI-spot assay developed for the detection of antigen presentation by lymphocytes. Our results showed that the positive rate of anti-H5 antibodies was much lower than that of anti-H9 antibodies among all the cohorts i.e. $86 \%$ chicken hawkers, $60 \%$ Flu patients and $37 \%$ healthy individuals showed immunity against avian influenza $\mathrm{H} 5$ strain included in local vaccine. Whereas, $93 \%$ chicken hawkers, $90 \%$ Flu patients and $79 \%$ healthy individuals revealed the presence of antibodies against $\mathrm{H} 7+\mathrm{H} 9$ local avian influenza vaccine. Representing that these avian influenza strains i.e. $\mathrm{H} 5, \mathrm{H} 7$ and $\mathrm{H} 9$ are also circulating being in our community and these individuals may acquire the sub lethal dose of viruses through stray birds or poultry (Figure 1). In addition, $\mathrm{H} 7$ and $\mathrm{H} 9$ avian influenza virus infection in poultry is usually asymptomatic and associated with virus shedding. Our finding highlights the potential risk of these avian influenza viruses to public health (Figure 1). These results also corroborate the findings of previous investigators, according to which all anti-H5-positive persons were involved in poultry business i.e. retailers or wholesalers, but antiH9-positive persons were identified in all study groups [17].

Chicken hawkers also showed more robust and diversified immune response than Flu patients and healthy individuals because strong intensity of spots indicated high titer of antibodies in their sera against avian influenza viruses (Figure 1). The qualitative analysis indicated that chicken hawkers may have developed high titer of antibodies due to the continuous exposure of sub lethal dose of avian viruses. But the repeated exposure to the virus, as could occur during the routine handling of viral infected chickens, may result in antibody response in the humans. Based on the conclusive understanding from these results, we also propose the dissemination cycle of influenza virus from migratory bird to domestic birds, poultry birds and humans as illustrated in Figure 5.

The postulate under which the next part of this study was done was that all the poultry associated persons might have same exposure level of influenza viruses, but presence of antibodies may be associated with their age, health conditions and their immune response. For this purpose lymphocytes were isolated and cell mediated immune response was determined from poultry related personals, healthy individuals and flu patient's by Eli-Spot assay and HLA of lymphocytes.

Human major histocompatibility complex (MHC) polymorphism contributes to the genetic diversity of the species and to the differences in susceptibility to diseases among genetically distinct groups, thus making it difficult for large-scale epidemics to occur [5]. The essential role of the HLA antigens lies in the self-recognition and thus defense against microorganisms [9]. 
Citation: Vahed H, Zafar MN, Jamil N (2017) Role of Humoral and Cell-Mediated Immune Responses against Influenza Infections. J Immuno Biol

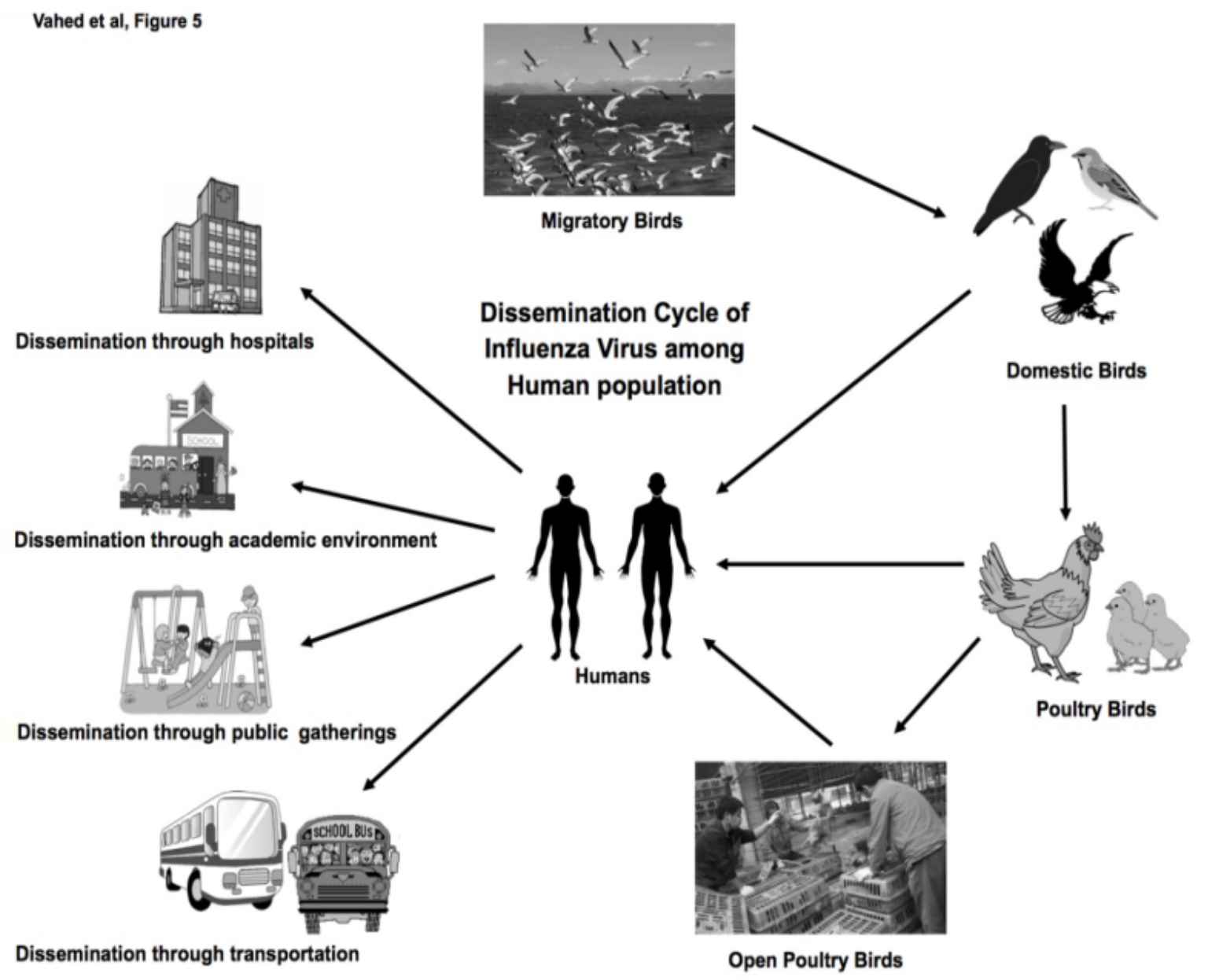

Figure 5: Dissemination cycle of influenza virus among human population. Based on the conclusive understanding from results, the dissemination cycle of influenza virus from migratory bird to domestic birds, poultry birds and humans was proposed in the following illustration.

The influence of gene polymorphism of MHC loci on the adaptive immunity levels of individuals including poultry hawkers $(n=13)$, Flu patients $(n=02)$ against influenza strains, was determined by genotyping of the HLA Class I and II including A, B DR and DQB1. The results indicated that the individuals with homozygous alleles of $\mathrm{DR}$ and DQB1 at the MHC loci are able to make a stronger humoral immune response than either heterozygote. This reveals that the presence of extreme genetic polymorphism found in $\mathrm{MHC}$ of humans are beneficial. The quantities of antibodies therefore represents how severely individuals have been infected the influenza viruses (Table 1). HLAs corresponding to MHC class II (DQ and DR) present antigens to the T-lymphocytes CD4+ and eventually stimulate the multiplication of T-helper cells, which in turn stimulate antibodyproducing B-cells to produce antibodies to that specific antigen. These $\mathrm{CD} 4+$ cells are necessary for long lasting effective CD8+ memory as well.

\begin{tabular}{|l|l|l|l|l|l|l|l|}
\hline \multirow{2}{*}{$\begin{array}{l}\text { Chicken } \\
\text { Serum }\end{array}$} & Hawkers & \multicolumn{2}{l}{ Vaccines (O.D) } & \multicolumn{2}{l|}{ MHC II LOCUS } & \multicolumn{2}{l|}{ MHC I LOCUS } \\
\cline { 2 - 9 } & H5 & H7 + H9 & Vaxigrip 2009/2010 & DR & DQB1 & A & B \\
\hline ChkHwk 1 & 0.374 & 0.377 & 0.456 & DR2(15), DR4 & DQ5(1), DQ7(3) & A3, A28(68) & B35, B22(55) \\
\hline ChkHwk 2 & 0.867 & 0.862 & 0.899 & DR2(15) & DQ6(1) & A2, A19(33) & B48 \\
\hline ChkHwk 3 & 0.361 & 0.345 & 0.372 & DR3(17), DR6(13.1) & DQ2, DQ6(1) & A1, A11 & B8, B22(55) \\
\hline ChkHwk 4 & 0.336 & 0.33 & 0.432 & DR2(15), DR6(13.1) & DQ6(1) & A1, A2 & B40(61), B15(63) \\
\hline
\end{tabular}


Page 7 of 8

\begin{tabular}{|l|l|l|l|l|l|l|l|}
\hline ChkHwk 5 & 0.853 & 0.85 & 0.985 & DR5(11) & DQ7(3) & A10(26), A19(32) & B7, B35 \\
\hline ChkHwk 6 & 0.355 & 0.371 & 0.475 & DR2(15), DR7 & DQ6(1), DQ9(3) & A1, A3 & B40(61), B46 \\
\hline ChkHwk 7 & 0.354 & 0.368 & 0.461 & DR3(17), DR3(18) & DQ6(1), DQ2 & A1, A10(26) & B8, B15(63) \\
\hline ChkHwk 8 & 0.396 & 0.417 & 0.477 & DR3(17), DR4 & DQ2, DQ8(3) & A1, A19(33) & B8, B15(62) \\
\hline ChkHwk 9 & 0.807 & 0.839 & 0.97 & DR2(15) & DQ6(1) & A9(24) & B35, B15(75) \\
\hline ChkHwk 10 & 0.799 & 0.877 & 0.936 & DR6(14.1) & DQ5(1) & A1, A11 & B35, B15(63) \\
\hline ChkHwk 11 & 0.453 & 0.468 & 0.523 & DR7 & DQ9(3), DQ2 & A9(24), A19(33) & B12(44), B17(57) \\
\hline ChkHwk 12 & 0.324 & 0.366 & 0.327 & DR7, DR5(11) & DQ7(3), DQ9(3) & A19(29), A28(68) & B22(55) \\
\hline ChkHwk 13 & 0.372 & 0.394 & 0.493 & DR2(15), DR4 & DQ6(1), DQ8(3) & A9(24), A11 & B15(62), B5(51) \\
\hline SN1 (Flu patient) & 0.342 & 0.337 & 0.363 & DR1404, DR5(11) & DQ5(1), DQ7(3) & A2, A11 & B35, B5(51) \\
\hline SN2 (Flu patient) & 0.846 & 0.849 & 0.956 & DR3(17) & DQ2 & A19(30), A19(33) & B13, B17(58) \\
\hline
\end{tabular}

Table I: HLA genotyping of cohorts of chicken hawker and flu patients related to influenza seropositive results in the study. HLA subtyping of the samples were performed by using CTS-PCR-SSP tray kit, containing 141 primer mixture sequences as described in materials and methods. The results indicated the HLA homozygosis at the MHC class II (Bold) for both DQB1 and DR alleles, including, poultry hawkers ( $\mathrm{n}=4$ ) and Flu patient $(n=1)$ showing a highly significant association of loci with the influenza infection (Table 1$)$ as having high level of antibodies against H5, H7, H9 and Vaxigrip 2009/2010 containing A/Brisbane/59/2007( H1N1)-souche, A/Brisbane/10/2007 (H2N2)-souche , B/Brisbane/60/2008souche. Whereas, homozygosis at HLA-class I loci A and B (Bold) had no effect on the rate of disease progression. These findings indicate an important role of the products of the class II loci in the immune response to influenza. This study demonstrates that MHC Class II alleles are majorly involved in the susceptibility and resistance to influenza infections.

The majority of the individuals were heterozygous at all HLA Loci showing a highly significant association of HLA homozygosis at the MHC class II loci with the influenza infection (Table 1). Homozygosis at HLA-class I loci A and B had no effect on the rate of disease progression. These findings indicate an important role of the products of the class II loci in the immune response to influenza (Table 1). This study demonstrates that MHC Class II alleles are majorly involved in the susceptibility and resistance to influenza infections. Such immunogenetical studies will help to develop an enhanced mechanistic understanding of the immune response to seasonal flu responses as well. These results are corroborated with the Nobel Prize winning discovery by Zinkernagel and Doherty that cellular immunity to viral infections was 'restricted' because of highly variable proteins of Major Histocompatibility Complex (MHC) brings the immunogenetics field in focus [18]. They demonstrated that the $\mathrm{T}$ lymphocytes recognize virus antigens that are displayed on host cells "in context of" the MHC proteins immediately suggest the idea that the genetic differences at loci encoding the MHC may modulate the intensities and effectiveness of host responses to the infections [19]. Similar results were also observed for different virus i.e. People in whom all the class II HLA alleles are heterozygous are the ones who most likely clear hepatitis B virus infections [14] while people with heterozygous class I alleles progress more slowly from HIV to an AIDS-defining illnesses and have lower mortality rates [14]. The converse scenario states that increased HLA homozygosity may contribute to increased susceptibility to the infections in populations that are genetically isolated. Overall these observations point towards a major role of classical HLA loci for determining the susceptibility to viral infections [14].

As mentioned in the previous literature, cell-mediated immunity to natural influenza infection is long lived and correlated with humoral immunity. The humoral response to influenza includes the development of neutralizing antibodies against the surface glycoprotein, hemagglutinin. This antibody response is seen at 4 to 7 weeks post-infection and declines slowly afterwards. Although the antibody response is very important in subsequent protection against infection, CD4+ and CD8+ T-cell responses also play a role $[20,21]$. Cytotoxic T-lymphocyte (CTL) responses are predominantly directed to internal viral proteins, the Nucleoprotein (NP) in particular, which is much more conserved than the surface hemagglutinin and neuraminidase glycoproteins. The preexisting CTL immunity against influenza virus may be of importance in the face of the current H5N1 pandemic threat; our knowledge of the cross-reactive nature of the human CTL response is limited. However, this cellular immunity has been shown to diminish over years [20].

There is also a report describing the presence of virus specific antigens on the surface of circulating blood lymphocytes of patients suffering from influenza [20]. Due to these facts, the Eli-Spot assay was also performed parallel for the detection of H5, H7 and H9 antigenspecific CD8+ cells in from the same individuals including chicken hawkers, flu patients and healthy individuals. Eli-Spot assay is sensitive and is suitable for the analysis of the aggregate CD8+ response to viralinfected lymphocytes. The examination of 50 lymphocytes samples revealed the positive spot for $100 \%$ lymphocytes for influenza virus subtype H9. Whereas $90 \%$ and $70 \%$ lymphocytes were positive for influenza virus subtype $\mathrm{H} 7$ and $\mathrm{H} 5$ respectively (Figure 4).

Interestingly it has been established from the results that all those individuals who were having antibodies against $\mathrm{H} 5, \mathrm{H} 7$ and $\mathrm{H} 9$ were positive for Eli-Spot assay. Might be these individuals did not had H5 infection but the CTL epitopes are cross reactive with other human influenza viruses. These results are concordance with the other studies, according to which, for most CTL epitopes, it was found that a vast majority of the $\mathrm{H} 5 \mathrm{~N} 1$ strains contained epitope sequences identical to those present in human influenza A viruses. This conservation of 
Citation: Vahed H, Zafar MN, Jamil N (2017) Role of Humoral and Cell-Mediated Immune Responses against Influenza Infections. J Immuno Biol 2: 117. doi:10.4172/2476-1966.1000117

Page 8 of 8

epitopes is responsible for the cross-reactive nature of CTL responses in humans against seasonal influenza virus.

It can be concluded that human cell-mediated immunity by T-cells, is long lived as compared with humoral immunity and the response displays a high degree of cross-reactivity among influenza viruses and could reduce morbidity and mortality during a pandemic caused by new evolving influenza virus strains.

\section{Acknowledgements}

The authors gratefully acknowledge use of the services and facilities of Sindh Institute of Transplantation, Karachi, Pakistan and Higher Education Commission, Pakistan for providing funds to complete this project.

\section{References}

1. Hubalek Z (2003) Emerging human infectious diseases: anthroponoses, zoonoses, and sapronoses. Emerg Infect Dis 9: 403-404.

2. Pedersden KA, Sadasiv EC, Chang PW, Yates VJ (1990) Detection of antibody to avian viruses in human populations. Epidemiol Infect 104: 519-525.

3. Dubois J, Terrier O, Rosa-Calatrava M (2014) Influenza viruses and mRNA splicing: doing more with less. MBio 5: e00070-e00114.

4. Mahmood MS, Ali S, Hussain I, Aslam A, Rafique A (2014) The development of hydropericardium syndrome vaccines. World's Poul Sci J 70: 355-364.

5. Alves C, Souza T, Meyer I, Toralles MB, Brites C (2006) Immunogenetics and infectious diseases: special reference to the mayor histocompatibility complex. Braz J Infect Dis 10: 122-131.

6. Singh N, Agrawal S, Rastogi AK (1997) Infectious diseases and immunity: special reference to major histocompatibility complex. Emerg Infect Dis 3: 41-49.

7. Hill AV (1998) The immunogenetics of human infectious diseases. Annu Rev Immunol 16: 593-617.

8. Konig R, Fleury S, Germain RN (1996) The structural basis of CD4-MHC class II interactions: coreceptor contributions to $\mathrm{T}$ cell receptor antigen recognition and oligomerization-dependent signal transduction. Curr Top Microbiol Immunol 205: 19-46.

9. Chaplin DD (2010) Overview of the immune response. J Allergy Clin Immunol 125: S03-S23.

10. Huang X, Ling H, Mao W, Ding X, Zhou Q, et al. (2009) Association of HLA-A, B, DRB1 alleles and haplotypes with HIV-1 infection in Chongqing, China. BMC Infect Dis 9: 201.

11. Carrington M, O'Brien SJ (2003) The influence of HLA genotype on AIDS. Annu Rev Med 54: 535-551.

12. Drukker M, Katz G, Urbach A, Schuldiner M, Markel G, et al. (2002) Characterization of the expression of MHC proteins in human embryonic stem cells. Proc Natl Acad Sci U S A 99: 9864-9869.

13. Jaratlerdsiri W, Deakin J, Godinez RM, Shan X, Peterson DG, et al. (2014) Comparative genome analyses reveal distinct structure in the saltwater crocodile MHC. PLoS One 9: e114631.

14. Blackwell JM, Jamieson SE, Burgner D (2009) HLA and infectious diseases. Clin Microbiol Rev 22: 370-385.

15. Davenport MP, Hill AV (1996) Reverse immunogenetics: from HLAdisease associations to vaccine candidates. Mol Med Today 2: 38-45.

16. Webster RG, Peiris M, Chen H, Guan Y (2006) H5N1 outbreaks and enzootic influenza. Emerg Infect Dis 12: 3-8.

17. Wang M, Fu CX, Zheng BJ (2009) Antibodies against H5 and H9 Avian Influenza among Poultry Workers in China. $\mathrm{N}$ Engl J Med 360: 2583-2584.

18. Cottingham MG, van Maurik A, Zago M, Newton AT, Anderson RJ, et al. (2006) Different levels of immunogenicity of two strains of Fowlpox virus as recombinant vaccine vectors eliciting T-cell responses in heterologous prime-boost vaccination strategies. Clin Vaccine Immunol 13: 747-757.

19. Lipsitch M, Bergstrom CT, Antia R (2003) Effect of human leukocyte antigen heterozygosity on infectious disease outcome: the need for allelespecific measures. BMC Med Genet 4: 2.

20. Baluch A, Humar A, Egli A, Gubbay J, Lisboa L, et al. (2011) Long term immune responses to pandemic influenza $A / \mathrm{H} 1 \mathrm{~N} 1$ infection in solid organ transplant recipients. PLoS One 6: e28627.

21. Stanekova Z, Vareckova E (2010) Conserved epitopes of influenza A virus inducing protective immunity and their prospects for universal vaccine development. Virol J 7: 351. 